\title{
Water-Immersible MEMS scanning mirror designed for wide-field fast- scanning photoacoustic microscopy
}

Junjie Yao, Chih-Hsien Huang, Catherine Martel, Konstantin I. Maslov, Lidai Wang, et al.

Junjie Yao, Chih-Hsien Huang, Catherine Martel, Konstantin I. Maslov, Lidai Wang, Joon-Mo Yang, Liang Gao, Gwendalyn Randolph, Jun Zou, Lihong V. Wang, "Water-Immersible MEMS scanning mirror designed for wide-field fast-scanning photoacoustic microscopy," Proc. SPIE 8581, Photons Plus Ultrasound: Imaging and Sensing 2013, 858127 (4 March 2013); doi: $10.1117 / 12.2005669$

SPIE. Event: SPIE BiOS, 2013, San Francisco, California, United States 


\title{
Water-Immersible MEMS Scanning Mirror Designed for Wide-field Fast-scanning Photoacoustic Microscopy
}

\author{
Junjie Yao ${ }^{1}$, Chih-Hsien Huang ${ }^{2}$, Catherine Martel ${ }^{3}$, Konstantin I. Maslov ${ }^{1}$, Lidai Wang ${ }^{1}$, Joon-Mo \\ Yang ${ }^{1}$, Liang Gao ${ }^{1}$, Gwendalyn Randolph ${ }^{3}$, Jun Zou ${ }^{2}$, Lihong V. Wang ${ }^{1 *}$ \\ ${ }^{1}$ Department of Biomedical Engineering, Washington University in St. Louis, \\ St. Louis, MO 63130, USA \\ ${ }^{2}$ Department of Electrical and Computer Engineering, Texas A\&M University, \\ College Station, TX 77843, USA \\ ${ }^{3}$ Department of Pathology and Immunology, Washington University School of Medicine, \\ St. Louis, MO 63110, USA
}

\begin{abstract}
By offering images with high spatial resolution and unique optical absorption contrast, optical-resolution photoacoustic microscopy (OR-PAM) has gained increasing attention in biomedical research. Recent developments in OR-PAM have improved its imaging speed, but have sacrificed either the detection sensitivity or field of view or both. We have developed a wide-field fast-scanning OR-PAM by using a water-immersible MEMS scanning mirror (MEMS-ORPAM). Made of silicon with a gold coating, the MEMS mirror plate can reflect both optical and acoustic beams. Because it uses an electromagnetic driving force, the whole MEMS scanning system can be submerged in water. In MEMS-ORPAM, the optical and acoustic beams are confocally configured and simultaneously steered, which ensures uniform detection sensitivity. A B-scan imaging speed as high as $400 \mathrm{~Hz}$ can be achieved over a $3 \mathrm{~mm}$ scanning range. A diffraction-limited lateral resolution of $2.4 \mu \mathrm{m}$ in water and a maximum imaging depth of $1.1 \mathrm{~mm}$ in soft tissue have been experimentally determined. Using the system, we imaged the flow dynamics of both red blood cells and carbon particles in a mouse ear in vivo. By using Evans blue dye as the contrast agent, we also imaged the flow dynamics of lymphatic vessels in a mouse tail in vivo. The results show that MEMS-OR-PAM could be a powerful tool for studying highly dynamic and time-sensitive biological phenomena.
\end{abstract}

Keywords: Optical-resolution photoacoustic microscopy, MEMS scanning mirror, blood flow dynamic imaging, lymphatic flow dynamic imaging, functional brain imaging

\section{INTRODUCTION}

As a major implementation of photoacoustic tomography, optical-resolution photoacoustic microscopy (OR-PAM) has been proven capable of anatomical, chemical, functional and metabolic imaging [1-4]. Several scanning mechanisms have been reported to accelerate the imaging speed of OR-PAM. These methods, however, lacked either wide scanning range or good detection sensitivity [5-7]. Here, we present a wide-field fast-scanning OR-PAM using a lab-made waterimmersible MEMS (i.e., microelectromechanical system) scanning mirror (MEMS-OR-PAM) [8].

\section{MEMS-OR-PAM SYSTEM}

As shown in Figure 1, a pulsed laser beam at $532 \mathrm{~nm}$ from an Nd:YVO4 laser is focused by a condenser lens, then spatially filtered by a pinhole. The filtered laser beam is focused by an optical objective lens. A beam combiner provides acoustic-optical coaxial alignment. The focused laser beam and the generated photoacoustic waves are both directed by a MEMS scanning mirror plate. The photoacoustic waves are then focused by an acoustic lens and detected by an

*Corresponding author: Lihong Wang, lhwang@wustl.edu. Phone: 1-314-935-6152

Photons Plus Ultrasound: Imaging and Sensing 2013, edited by Alexander A. Oraevsky, Lihong V. Wang, Proc. of SPIE Vol. 8581, 858127 - (C) 2013 SPIE · CCC code: 1605-7422/13/\$18 · doi: 10.1117/12.2005669 
ultrasonic transducer. Driven by electromagnetic force, the whole MEMS scanning mirror can be submerged in water (Figure 2). Volumetric imaging is provided by fast angular scanning of the MEMS mirror along the $x$-axis and slow linear step-motor scanning of the sample along the $y$-axis. A $2.4 \mu \mathrm{m}$ diffraction-limited lateral resolution and $26 \mu \mathrm{m}$ axial resolution have been achieved, with a maximum penetration depth of $1.1 \mathrm{~mm}$ in biological tissue. The maximum in-focus scanning range is $\sim 3.0 \mathrm{~mm}$ along the $x$-axis, with a cross-sectional frame rate of $400 \mathrm{~Hz}$. By scanning simultaneously both the excitation laser beam and resultant photoacoustic beam, MEMS-OR-PAM maintains confocal alignment and high detection sensitivity over a large field of view $(\sim 3.0 \mathrm{~mm} \times 4.5 \mathrm{~mm})$.

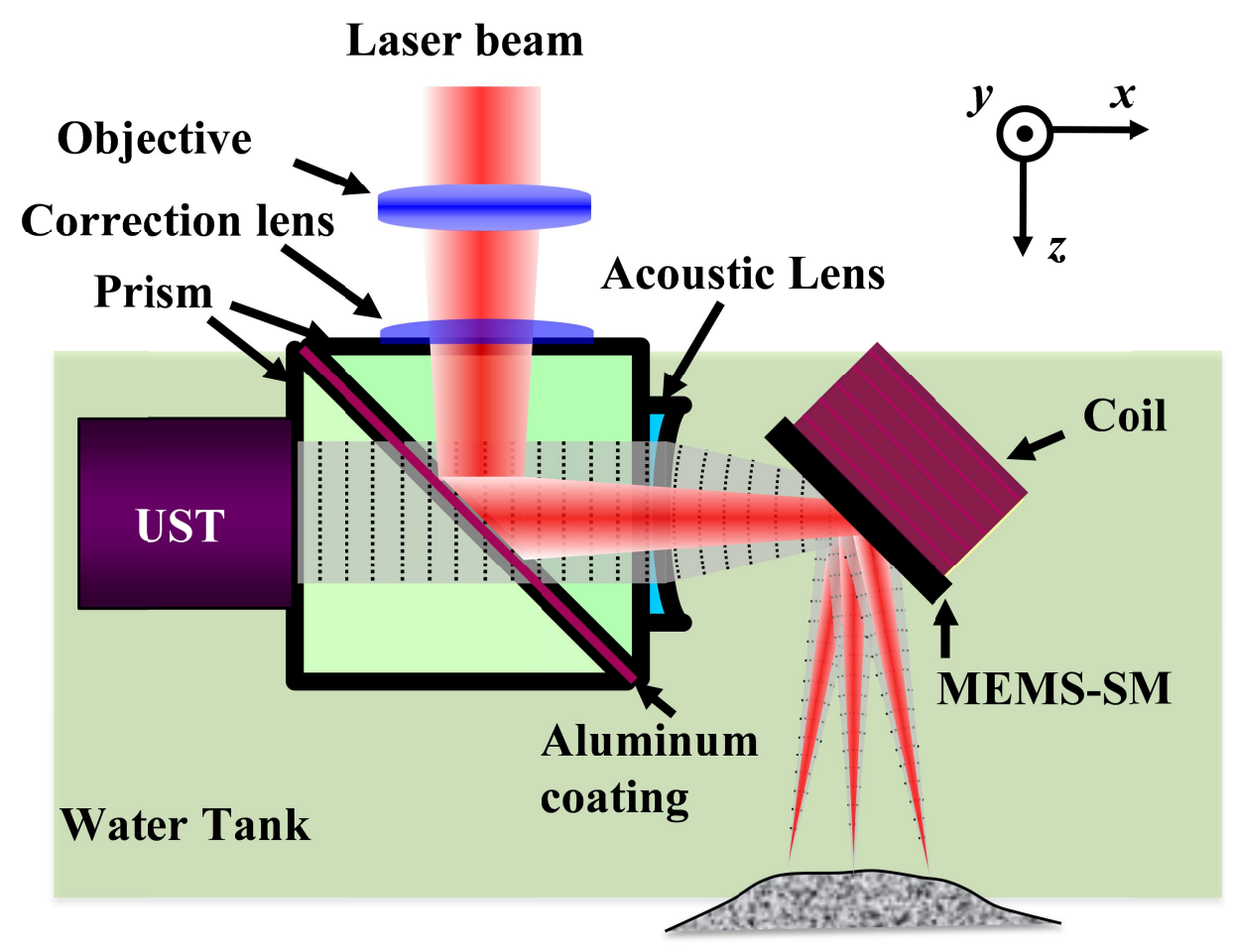

Figure 1. Schematic of MEMS-OR-PAM system.

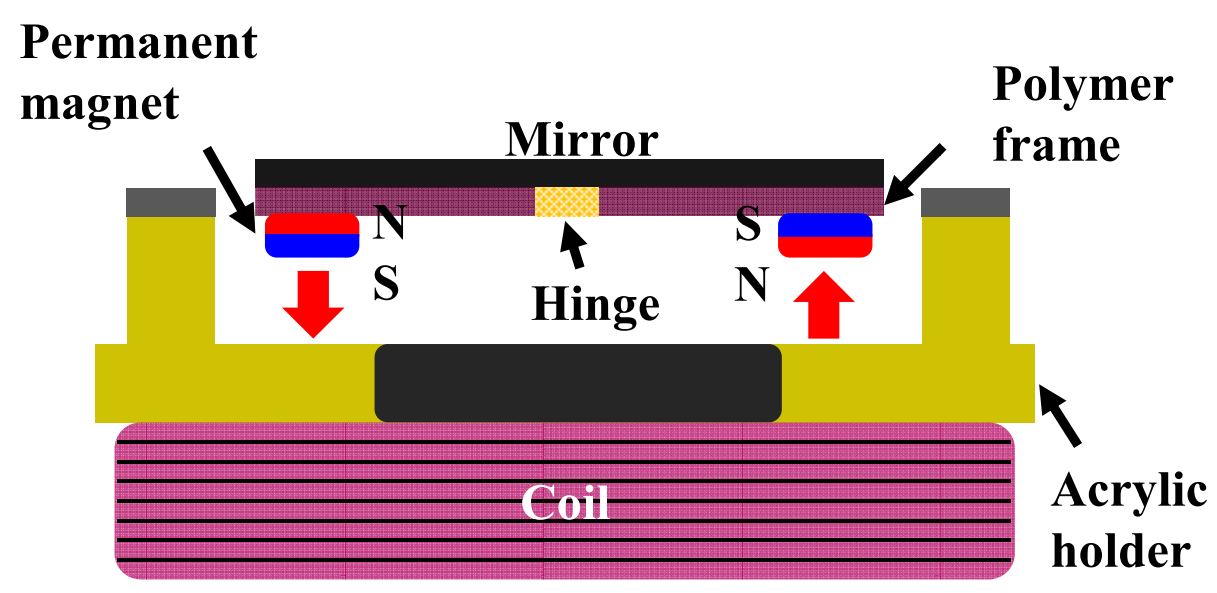

Figure 2. Schematic of water immersible MEMS scanning mirror. 


\section{BLOOD FLOW IMAGING IN VIVO}

Red blood cell (RBC) flow in a nude mouse ear was imaged in vivo by MEMS-OR-PAM to demonstrate its high-speed imaging capability. As shown in Figure 3, the mouse ear was repeatedly scanned with a volumetric imaging speed of 1 $\mathrm{Hz}$ (a 2D B-scan rate of $400 \mathrm{~Hz}$ ), which is about 400 times faster than the second-generation OR-PAM system and 20 times faster than the most recent voice-coil based OR-PAM scanner for the same scanning range. The spatial resolution is sufficient for resolving the capillary beds, and the imaging speed enables the mapping of the RBC movements in small vessels. In addition, intravascular transport of an exogenous contrast agent was also explored using the system. We injected carbon particles with $\sim 6 \mu \mathrm{m}$ diameters into the blood stream of the nude mouse via its tail vein. A $40-\mu \mathrm{m}$ diameter vein was repeatedly imaged with a volumetric frame rate of $4 \mathrm{~Hz}$. A low pulse energy of $10 \mathrm{~nJ}$ was used to image only the particles, which are much more absorbing than hemoglobin.

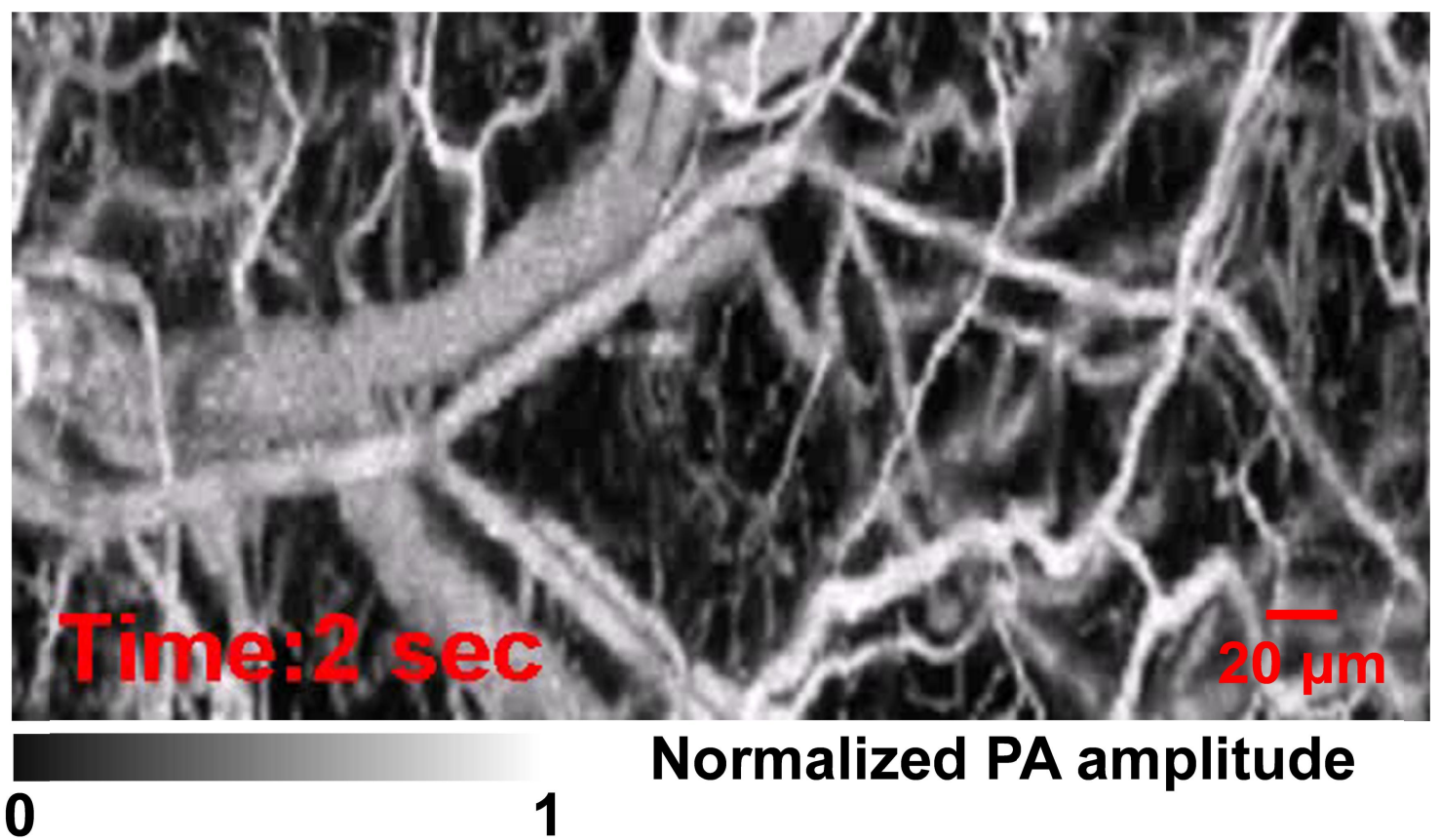

Figure 3. MEMS-OR-PAM of blood flow dynamics in a mouse ear.

\section{FUNCTIONAL MOUSE BRAIN IMAGING.}

High-performance functional brain imaging is extremely important to both fundamental neuroscience and clinical neurology. Nevertheless, none of the common brain imaging techniques (e.g., fMRI, PET and DOT) have achieved both high spatial and temporal resolutions simultaneously, which is critical when imaging complex transient events such as epileptic seizures. Here, as a demonstration, MEMS-OR-PAM was used to image cortical responses in the mouse brain evoked by electrical stimulation with a volumetric frame rate of $1 \mathrm{~Hz}$. 
(a)

(c)
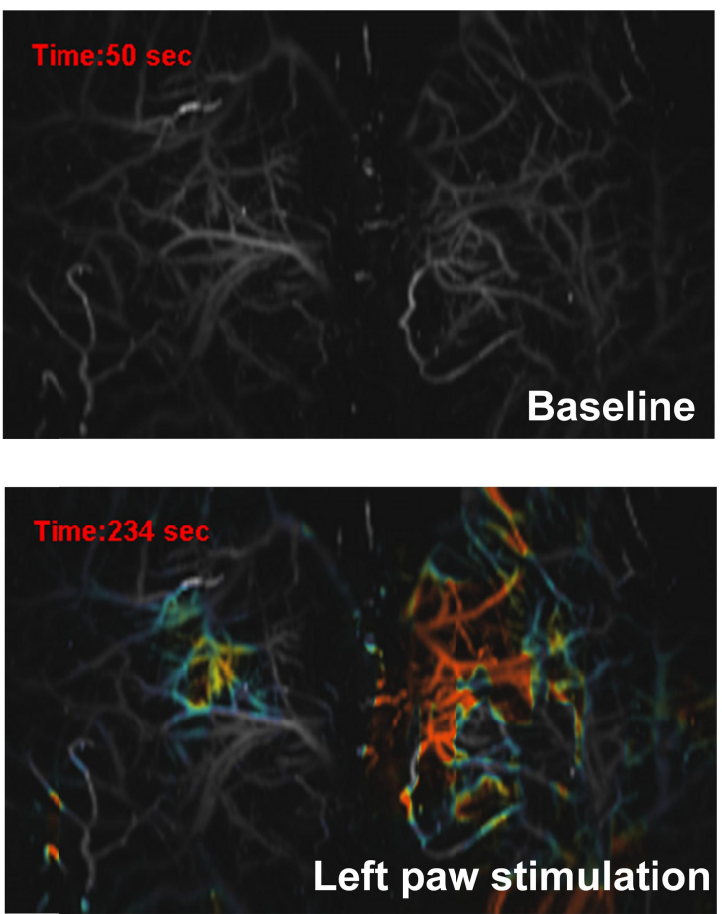

0 Relative PA amplitude 5 change (\%) (b)

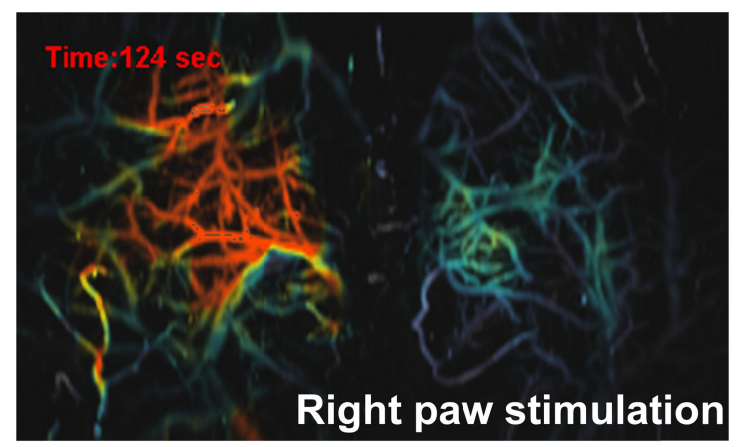

(d)

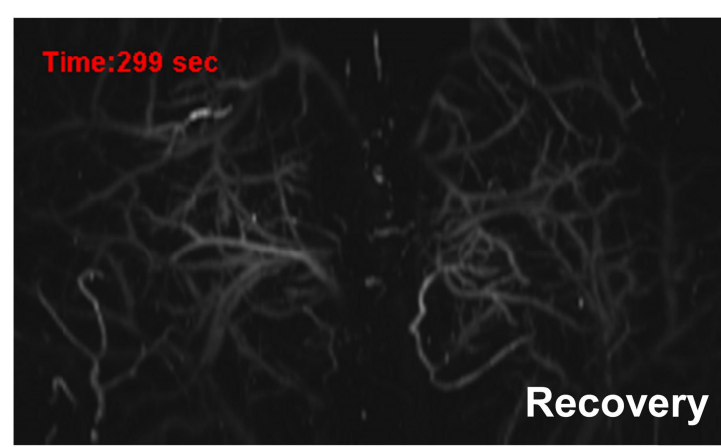

$500 \mu \mathrm{m}$

Figure 4. MEMS-OR-PAM of mouse brain responses to electrical stimulations on the hind paws.

Figure 4a shows a PAM image of the mouse brain, where the dense cortical vasculature can be clearly observed. Upon right paw stimulation, the relative PA amplitude change in the somatosensory region of the left hemisphere started to increase and peaked at the end of the stimulation (Figure 4b). Upon left paw stimulation, the relative PA amplitude change in the somatosensory region of the right hemisphere started to increase and peaked at the end of the stimulation (Figure 4c). The increase in PA signal amplitudes in contralateral hemispheres reflected the elevated neural activity evoked by the stimulations. The brain responses in the ipsilateral hemispheres consistently showed a similar trend to that of the contralateral hemispheres, but a weaker magnitude and a longer time lag. This observation may indicate cross talk between the two hemispheres of the brain. We also observed similar signal increases in the sagittal sinus region under both stimulations, which may be explained by the fact that the sagittal sinus drains blood from both hemispheres.

\section{FUNCTIONAL LYMPHATIC FLOW DYNAMIC IMAGING.}

Functional lymphatic vessel imaging is important in immunology studies and cancer research. Here, using MEMS-ORPAM, we preformed real-time lymphatic flow dynamic imaging in a mouse tail. First, the pumping dynamics of Evans blue dye in the superficial lymphatic vessels were imaged with a volumetric frame rate of $4 \mathrm{~Hz}$. The pumping feature of the superficial lymphatic vessels was clearly observed, which was believed to be the first time in mice. Then the deeper collective lymphatic vessels were also imaged with the Evans blue as the contrast agent. The time course of the collective lymphatic vessel showed that the lymphatic flow had a speed of $\sim 0.1 \mathrm{~mm} / \mathrm{s}$ (Figure 5). The flow direction can be determined based on the time lags of the time courses at different positions along the vessel. In addition, based on the temporal information, the lymphatic vessel can be separated from the blood vessels, as shown in Figure 5. 

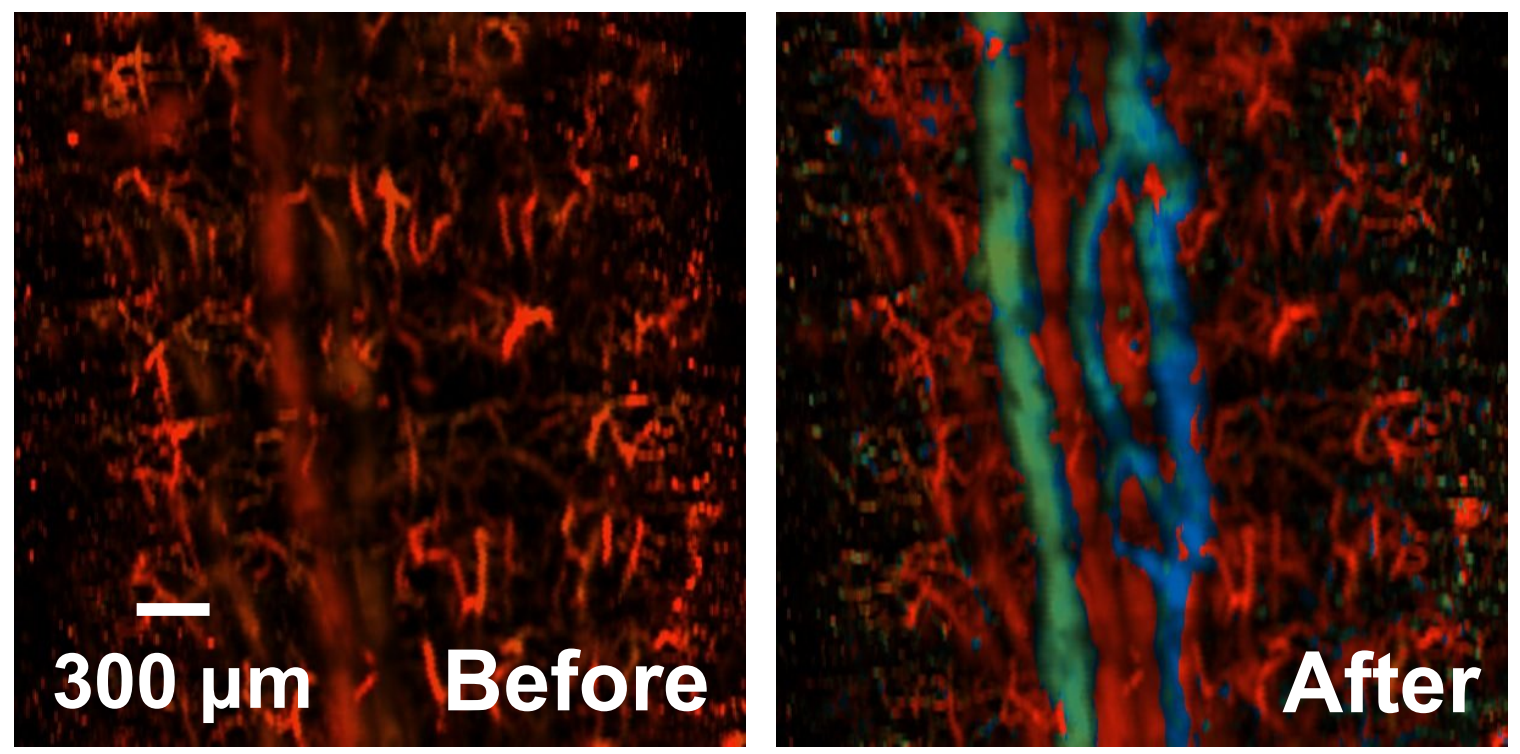

Figure 5. MEMS-OR-PAM of lymphatic flow dynamics in a mouse tail before and after the injection of Evans blue dye. The blood vessels are shown in red, while the lymphatic vessels are shown in blue.

\section{CONCLUSIONS}

In summary, we have developed a MEMS-OR-PAM system that can dramatically improve the imaging speed while maintaining the confocal alignment of the optical and acoustic beams over a wide field of view. A laser system with higher repetition rate or an intensity-modulated continuous wave laser can further improve the imaging speed while maintaining the high resolution and wide scanning range. By employing a dual-wavelength laser source, MEMS-ORPAM is intrinsically capable of label-free measurements of oxygen saturation and oxygen metabolism.

\section{ACKNOWLEDGEMENTS}

The authors would like to thank Prof. James Ballard for manuscript editing. We also appreciate technical assistance and useful discussions with Yan Liu. This research was supported by the National Institutes of Health Grants DP1 EB016986 (NIH Director's Pioneer Award), R01 EB008085, R01 CA134539, U54 CA136398, R01 CA157277, R01 CA159959. L.V.W. has a financial interest in Microphotoacoustics, Inc. and Endra, Inc., which, however, did not support this work.

\section{REFERENCES}

[1] Wang, L. H. V. and Hu, S., "Photoacoustic Tomography: In Vivo Imaging from Organelles to Organs," Science. 335(6075), 1458-1462 (2012).

[2] Yao, J., et al., "In vivo photoacoustic imaging of transverse blood flow by using Doppler broadening of bandwidth," Optics Letters. 35(9), 1419-1221 (2010).

[3] Yao, J., et al., "Label-free oxygen-metabolic photoacoustic microscopy in vivo," Journal of Biomedical Optics. 16(7), 076003 (2011).

[4] Yao, J. and Wang, L. V., "Photoacoustic tomography: fundamentals, advances and prospects," Contrast Media Mol Imaging. 6(5), 332-345 (2011).

[5] Wang, L. D., et al., "Fast voice-coil scanning optical-resolution photoacoustic microscopy," Optics Letters. 36(2), 139-141 (2011).

[6] Rao, B., et al., "Real-time four-dimensional optical-resolution photoacoustic microscopy with Au nanoparticleassisted subdiffraction-limit resolution," Optics Letters. 36(7), 1137-1139 (2011). 
[7] Xie, Z. X., et al., "Laser-scanning optical-resolution photoacoustic microscopy," Optics Letters. 34(12), 17711773 (2009).

[8] Yao, J., et al., "Wide-field fast-scanning photoacoustic microscopy based on a water-immersible MEMS scanning mirror," Journal of Biomedical Optics. 17(8), 080505 (2012).

Proc. of SPIE Vol. $8581858127-6$

Downloaded From: https://www.spiedigitallibrary.org/conference-proceedings-of-spie on 9/5/2018 Terms of Use: https://www.spiedigitallibrary.org/terms-of-use 